Perinatology pISSN 2508-4887 • elSSN 2508-4895
Original article

Perinatology Vol. 31, No. 4, December, 2020

https://doi.org/10.14734/PN.2020.31.4.172

\title{
Clinical Experiences of Amplitude-integrat- ed Electroencephalographic Monitoring in Neonatal Intensive Care Unit
}

\section{Sangeun Lee, MD', SuJin Choi, MD', Yun Jeong Lee, $\mathrm{MD}^{1}$, Jisook Kim, MD', Sook-Hyun Park, MD², Eun Joo Lee, MD ${ }^{1}$}

'Department of Pediatrics, Kyungpook National University Medical Center, ${ }^{2}$ Department of Pediatrics, Kyungpook National University Children's Hospital, Kyungpook National University School of Medicine, Daegu, Korea

Received: 13 January 2020

Revised: 26 March 2020

Accepted: 10 July 2020

Correspondence to

Eun Joo Lee, MD

Department of Pediatrics, Kyungpook National University Medical Center, Kyungpook National University School of Medicine, 130 Dongdeok-ro, Jung-gu, Daegu 41944, Korea

Tel: +82-53-200-5704

Fax: +82-53-425-6683

E-mail:pshmom00@gmail.com

Copyright@ 2020 by The Korean Society of Perinatology

This is an Open Access article distributed under the terms of the Creative Commons Attribution Non-Commercial License (http://creativecommons.org/ license/by-nc/4.0/), which permits unrestricted non-commercial use, distribution, and reproduction in any medium, provided that the original work is properly cited.
Objective: The purpose of this study was to analyze the clinical experience of amplitude-integrated electroencephalogram (aEEG) in the neonatal intensive care unit (NICU) and to evaluate the usefulness of the aEEG and to determine whether an EEG could help to identify high risk infants with later epilepsy.

Methods: Clinical data of 200 newborn infants admitted to the NICU and monitored with aEEG were reviewed retrospectively. A single- or two-channel aEEG (electrode placement P3-P4 for single, C3-C4 and P3-P4 for two) was recorded continuously by using gold cups. Background activity was assessed based on voltage and pattern recognition methods. To assess for differences in later epilepsy among infants with abnormal versus normal results, chi-square test was employed with odd ratio.

Results: Overall, 200 newborn infants were included. About half showed abnormal findings on aEEG monitoring with $34.5 \%$ abnormal background activity, 30.0\% abnormal cyclicity, and 30.0\% with seizures. The odd ratio for an abnormal trace on aEEG to predict later epilepsy was 7.9 (95\% confidence interval; 2.8-22.0; $P<0.001)$.

Conclusion: aEEG monitoring is useful for cerebral monitoring in NICU. aEEG monitoring enabled to assess the cerebral integrity of infants by measuring background activities and detecting seizures and help to identify high-risk infant for later epilepsy.

Key Words: Brain monitoring, Electroencephalogram, Newborns

\section{Introduction}

The amplitude-integrated electroencephalogram (aEEG) has been used for monitoring cerebral integrity since it was first introduced in the late 1960s by Maynard et al. ${ }^{1}$ at the London Hospital. aEEG began to be used in newborn infants since the $1980 \mathrm{~s} .{ }^{2,3}$ With the development of digital technology during the 2000s, raw EEG tracing was also displayed simultaneously with the aEEG tracing. ${ }^{4}$ aEEG is now a standard device for cerebral monitoring in neonatal intensive care unit (NICU) worldwide.

Of note, conventional electroencephalogram (cEEG) is the clinical standard for brain monitoring. ${ }^{4}$ However, cEEG may not be always available on even emergent basis. The need for a trained cEEG technologist for examination and the expertise for reading limits the use of cEEG in NICU. ${ }^{5}$ Newborn infants, including premature babies are vulnerable to brain damage and have a high risk of seizures; therefore cerebral function monitoring should be available in NICU. ${ }^{6,7}$ The emergence of aEEG has provided a complementary method that is easily accessible and applied regardless of expertise and allows long-term real-time monitoring. ${ }^{4.5}$ It also has been used as routine monitoring in many high level NICUs in Korea since 2008; nevertheless, there are few reports on the clinical experiences of aEEG monitoring. Therefore, the purpose of this study was to review our clinical experience, to analyze the potential of aEEG in the NICU and to determine whether aEEG could help identify infants at high risk for later epilepsy. 


\section{Methods}

Total of 3,165 newborn infants were admitted to the NICU at Kyungpook National University Hospital (n=788) and Kyungpook Children's Hospital ( $n=2,370)$ between March 2014 and December 2018. Both centers are level III NICUs in Daegu, Korea. Of these, aEEG monitoring was performed in 200 newborn infants (46 and 154, respectively) for monitoring seizures or background activities. The study was approved by the authors' institutional ethical committee (approval number: KNUCH 201911-004, 2020-01-041-001), and the requirement for written informed consent was waived as this was a de-identified, dataonly study.

By reviewing medical records, we collected clinical characteristics including gestational age, gender, birth weight, mode of delivery, 1-minute and 5-minutes Apgar scores and detailed postnatal history and clinically indicated neurologic testing results (e.g., brain ultrasonography (USG), brain magnetic resonance imaging (MRI), and cEEG retrospectively. Abnormal brain USG was defined in this study as grade III-IV intraventricular hemorrhage (IVH) or severe white matter damage. ${ }^{8,9}$ Brain MRI scans were considered abnormal if it showed evidence of developmental malformation, grade III or IV IVH or infarct, abnormal signal intensities within the basal ganglia, thalamus, internal capsule, subcortical white matter or cortex. The time of the events, the reason for the aEEG monitoring, and the underlying causes for events were evaluated based on the records. Cerebral causes were defined as seizure or encephalopathy while noncerebral causes were defined as nonconvulsive acute paroxysmal events (APE) or other systemic illness. APE was defined as clinically nonconvulsive and no specific findings on cerebral evaluation. ${ }^{10}$ If the infant has documented seizure, but the cause was not clear, and there were no specific findings in the evaluation, and no further seizures, the etiology was classified as unidentified, which was presumed as benign seizures. To determine whether aEEG can identify high-risk infant, we reviewed the presence of later epilepsy during follow up period, which was defined as taking antiepileptic medications for more than a year beyond neonatal period or having epilepsy diagnosis by neurologist.

The aEEG recordings were performed with the Olympic Brainz Monitor (Natus Medical Incorporated, San Carlos, CA, USA). A single- or two-channel aEEG (electrode placement parietal
[P3-P4] for single, central-parietal [C3-C4 and P3-P4] for two according to the international 10-20 system) was recorded continuously by using gold cups. aEEG monitoring had performed with single channel before but 2 channel monitoring has been performed routinely since March 2016. Most of the recordings were performed immediately after the event was recognized by the medical staff, the recording within the initial 24 hours were used for the assessment of the background activities. In the case of seizures, it was read as abnormal when confirmed through the whole aEEG monitoring. The background activities were assessed based on voltage and pattern recognition methods ${ }^{11-13}$ : continuous, discontinuous, burst suppression, low voltage and isoelectric trace. Cyclicity was classified as being absent, immature and mature.11 Electrographic seizures in the aEEG are defined as runs of rhythmic activities that initially increases in frequency and amplitude, reaches a maximum and decrease over a time period lasting at least 10 seconds. ${ }^{14}$ The recording was interpreted as abnormal if the infants showed the abnormal background activities or inappropriate cyclicity for gestational age, or the electrical seizures during the recording. Even premature infants with discontinuous background activities were read as normal if appropriate for the gestational age. The aEEG recordings were analyzed by two experienced investigators who were blinded to clinical information (Drs. Lee and Lee). Both have experience as neurologists of over 5 years and are competent at cEEG interpretation.

Analyses were performed using IBM SPSS Statistics ver. 25.0 (IBM Co., Armonk, NY, USA). Data for normally distributed continuous variables were expressed as means and standard deviations. For non-normally distributed variables were expressed as median and interquartile ranges. To assess for differences in later epilepsy among infants with abnormal versus normal results, the chi-square test was employed with odd ratio. A $P$-value of 0.05 was taken to be statistically significant.

\section{Results}

A total of 200 infants who underwent aEEG were enrolled in the study. Table 1 shows the demographic and clinical profile of enrolled infants. Sixty-seven preterm neonates (33.5\%) and 133 term infants (66.5\%) were included in the study. 
The purpose of cerebral monitoring is shown in Table 2, with $46.5 \%$ of recordings for monitoring seizures, $35.5 \%$ of the recording for monitoring background activities in infants who were suspected of having encephalopathy. The remainder was about $18.0 \%$ to discriminate seizures or encephalopathy for apnea. About half showed abnormal findings on aEEG monitoring, with 34.5\% abnormal background activity, 30.0\% abnormal cyclicity, and $30.0 \%$ seizures. In some cases, cEEG, brain USG, and brain MRI were not performed because the patient was seriously ill, died or did not need to be examined. Therefore, the number of aEEG trials was higher than that reported in other studies.

Table 3 shows the etiology of events (seizures, apnea, or encephalopathic features). About two-thirds were cerebral insults, which were manifested as seizures or encephalopathy. Among cerebral events, hypoxic-ischemic encephalopathy accounted for the largest number of infants. About one-third was considered to be other systemic illness such as sepsis and apnea of prematurity with normal aEEG tracing.

Seizures were observed in 119 of total 200 infants. Table 4 shows the phenotype of documented seizures. About a quarter did not confirm seizures. Clonic seizures were most frequently observed, and in $10.5 \%$, only electrical seizures were observed without clinical seizure (electro-clinical dissociation).

Table 5 lists the results of brain examination and the prediction of later epilepsy/later epilepsy or death. The outcomes are available for 169 (21 died and 10 were lost to follow up). The median

Table 1. Clinical Characteristics of Newborn Infants

\begin{tabular}{lc}
\hline Variable & Value \\
\hline Gestational age (weeks) & $38.0(35.3-39.7)$ \\
\hline Birth weight (g) & $2,925.0(2,195.0-3,345.0)$ \\
Sex & $112(56.0)$ \\
Male & $88(44.0)$ \\
Female & \\
Delivery & $92(46.0)$ \\
NSVD & $108(54.0)$ \\
C-sec & $84(42.0)$ \\
Inborn & $7.0(4.0-8.0)$ \\
1 minute Apgar score & $8.0(7.0-9.0)$ \\
5 minutes Apgar score & $33.0(30.0-36.0)$ \\
\hline Maternal age (years) &
\end{tabular}

Values are presented as number (\%) or median (interquartile range).

Abbreviations: NSVD, normal spontaneous vaginal delivery; C-sec, cesarean section.
Table 2. Results of aEEG Monitoring and Other Modalities

\begin{tabular}{|c|c|}
\hline Variables & Value \\
\hline \multicolumn{2}{|l|}{ aEEG monitoring } \\
\hline \multicolumn{2}{|l|}{ Aim of study } \\
\hline Background monitoring & $71(35.5)$ \\
\hline Seizure monitoring & $93(46.5)$ \\
\hline Apnea monitoring & $36(18.0)$ \\
\hline \multicolumn{2}{|l|}{ Number of channels } \\
\hline 1 channel & $47(23.5)$ \\
\hline 2 channels & $153(76.5)$ \\
\hline Recording time (hours) & $66.8(18.2-134.2)$ \\
\hline Event onset age (days) & $5(2-13)$ \\
\hline \multicolumn{2}{|l|}{ Result of aEEG monitoring } \\
\hline Normal & $100(50.0)$ \\
\hline Abnormal & $100(50.0)$ \\
\hline \multicolumn{2}{|l|}{ Abnormal aEEG monitoring } \\
\hline Abnormal background & $69(34.5)$ \\
\hline Abnormal cyclicity & $70(35.0)$ \\
\hline Seizure & $74(37.0)$ \\
\hline \multicolumn{2}{|c|}{ Background activities of aEEG monitoring } \\
\hline Continuous & $123(61.5)$ \\
\hline Discontinuous & $30(15.0)$ \\
\hline Burst suppression & $29(14.5)$ \\
\hline Low voltage & $10(5.0)$ \\
\hline Isoelectric & $8(4.0)$ \\
\hline \multicolumn{2}{|l|}{ Other modalities } \\
\hline \multicolumn{2}{|l|}{ EEG } \\
\hline Normal & $89(44.5)$ \\
\hline Abnormal & $40(20.0)$ \\
\hline Not done & $71(35.5)$ \\
\hline \multicolumn{2}{|l|}{ Brain USG } \\
\hline None or mild injury & $96(48.0)$ \\
\hline Moderate to severe injury & $55(27.5)$ \\
\hline Not done & $49(24.5)$ \\
\hline \multicolumn{2}{|l|}{ Brain MRI } \\
\hline Normal & $52(26.0)$ \\
\hline Abnormal & $58(29.0)$ \\
\hline Not done & $90(45.0)$ \\
\hline \multicolumn{2}{|l|}{ Brain USG or Brain MRI } \\
\hline Normal & $93(46.5)$ \\
\hline Abnormal & $80(40.0)$ \\
\hline Not done & 27 (13.5) \\
\hline
\end{tabular}

Values are presented as number (\%) or median (interquartile range). Abbreviations: $\mathrm{aEEG}$, amplitude-integrated electroencephalography; $\mathrm{EEG}$, electroencephalography; brain USG, brain ultrasonography; brain MRI, brain magnetic resonance imaging. 
Table 3. Underlying Etiologies of Events

\begin{tabular}{|c|c|}
\hline Etiology & Value \\
\hline With seizures or encephalopathy & $124(62.0)$ \\
\hline HIE & $45(36.3)$ \\
\hline Vascular & $15(12.1)$ \\
\hline $\mathrm{IVH} / \mathrm{ICH}$ & $12(9.7)$ \\
\hline Infarction & $2(1.6)$ \\
\hline Subgaleal hemorrhage & $1(0.8)$ \\
\hline Metabolic problems & $14(11.3)$ \\
\hline Hypocalcemia & $11(8.9)$ \\
\hline Hypoglycemia & $2(1.6)$ \\
\hline Hyponatremia & $1(0.8)$ \\
\hline CNS infection & $10(8.1)$ \\
\hline Rota encephalopathy & $5(4.0)$ \\
\hline Group B streptococcus & $4(3.2)$ \\
\hline Cytomegalovirus infection & $1(0.8)$ \\
\hline Inborn errors of metabolism & $6(4.8)$ \\
\hline Neonatal epileptic syndrome & $6(4.8)$ \\
\hline Chromosome/Syndromic & $5(4.0)$ \\
\hline Edward syndrome & $3(2.4)$ \\
\hline Prader Willi syndrome & $1(0.8)$ \\
\hline Spinal muscular atrophy & $1(0.8)$ \\
\hline Structural & $3(2.4)$ \\
\hline Neurocutaneous syndrome & $2(1.6)$ \\
\hline Unidentified & $18(14.5)$ \\
\hline Neither seizures nor encephalopathy & $76(38.0)$ \\
\hline Acute paroxysmal event & $11(5.5)$ \\
\hline Other systemic illness & $47(23.5)$ \\
\hline Sepsis or other infection & $23(11.5)$ \\
\hline Chromosomal/Syndromic & $8(3.5)$ \\
\hline Apnea of prematurity & $7(4.0)$ \\
\hline Feeding intolerance & $3(1.5)$ \\
\hline RDS/PPHN & $3(1.5)$ \\
\hline Heart disease (postsurgery) & $3(1.5)$ \\
\hline Just for monitoring & $15(7.5)$ \\
\hline Hypocalcemia & $6(3.0)$ \\
\hline Suspected hypoxic event & $4(2.0)$ \\
\hline Hyperbilirubinemia & $4(2.0)$ \\
\hline Inborn errors of metabolism & $1(0.005)$ \\
\hline
\end{tabular}

Values are presented as number (\%).

Abbreviations: HIE, hypoxic-ischemic-encephalopathy; IVH, intraventricular he morrhage; $\mathrm{ICH}$, intracranial hemorrhage; CNS, central nervous system; RDS, respiratory distress syndrome; PPHN, persistent pulmonary hypertension of newborn. follow-up days (interquartile range) were 383.0 (44.5-550.5). The odd ratio (OR) for an abnormal trace on aEEG to predict later epilepsy was 7.9 (95\% confidence interval [CI] 2.8-22.0, $P<0.001)$. In other words, as in the case of brain USG, brain MRI and cEEG abnormalities, the risk of later epilepsy was shown to be elevated. The odd ratio for later epilepsy or death much increased with abnormal aEEG compared with normal aEEG 15.7 (95\% CI 5.86-42.1, $P<0.001)$.

Table 4. Phenotypes of Documented Seizures ( $n=119)$

\begin{tabular}{lc}
\hline Seizure phenotype & Value \\
\hline Documented seizure & $119(59.5)$ \\
Clonic & $37(18.5)$ \\
Subtle & $23(11.5)$ \\
\hline Tonic & $19(9.5)$ \\
Myoclonic & $6(3.0)$ \\
Generalized tonic-clonic & $5(2.5)$ \\
Spasms & $3(1.5)$ \\
\hline Electrical seizure only & $26(13.0)$ \\
\hline
\end{tabular}

Values are presented as number (\%).

Table 5. Risk Analysis for the Later Epilepsy and Later Epilepsy or Death According to the Modalities

\begin{tabular}{|cccccc}
\hline Modality (n) & Normal & Abnormal & Odds ratio & $95 \% \mathrm{Cl}$ & $P$-value \\
\hline Later epilepsy & & & & & \\
\hline aEEG (169) & $5(5.3)$ & $23(30.7)$ & 7.9 & $2.8-22.0$ & $<0.001$ \\
\hline BG & $12(9.8)$ & $16(34.8)$ & 4.9 & $2.1-11.5$ & $<0.001$ \\
\hline Cyclicity & $13(10.7)$ & $15(31.5)$ & 3.9 & $1.7-9.1$ & 0.001 \\
\hline Seizures & $9(7.8)$ & $19(35.2)$ & 6.4 & $2.7-15.4$ & $<0.001$ \\
\hline EEG (118) & $9(10.8)$ & $16(45.7)$ & 6.9 & $2.7-18.1$ & $<0.001$ \\
\hline Brain USG (128) & $6(6.7)$ & $12(32.0)$ & 6.5 & $2.2-18.9$ & $<0.001$ \\
\hline Brain MRI (104) & $4(8.3)$ & $17(30.4)$ & 4.8 & $1.5-15.5$ & 0.005 \\
\hline Later epilepsy or death & & & & & \\
\hline aEEG (190) & $5(5.3)$ & $45(46.9)$ & 15.7 & $5.86-42.1$ & $<0.001$ \\
\hline BG & $13(10.5)$ & $37(56.1)$ & 10.9 & $5.1-23.1$ & $<0.001$ \\
\hline Cyclicity & $14(11.4)$ & $36(53.7)$ & 9 & $4.3-18.9$ & 0.001 \\
\hline Seizures & $13(10.9)$ & $37(52.1)$ & 8.9 & $4.2-18.6$ & $<0.001$ \\
\hline EEG (122) & $10(11.9)$ & $19(50.0)$ & 7.4 & $3.0-18.5$ & $<0.001$ \\
\hline Brain USG (144) & $8(8.7)$ & $27(51.9)$ & 11.3 & $4.6-28.0$ & $<0.001$ \\
\hline Brain MRI (105) & $5(10.2)$ & $17(30.4)$ & 3.8 & $1.2-11.4$ & 0.011 \\
\hline Ga & & & & \\
\hline
\end{tabular}

Values are presented as number (\%).

Abbreviations: $\mathrm{Cl}$, confidence interval; aEEG, amplitude-integrated electroencephalography; BG, background activities; EEG, electroencephalography; brain USG, brain ultrasonography; brain MRI, brain magnetic resonance imaging. 


\section{Discussion}

Since aEEG has been applied to NICU since the 1980s, many advances have been made, and nowadays, early aEEG findings are helpful in identifying newborns who benefit from cooling and predicting prognosis in HIE patients, ${ }^{11,15-18}$ and in recent years, many studies have shown that aEEG is helpful in predicting prognosis in premature infants. ${ }^{19-21}$ Since the best advantage of aEEG is that it can be continuously monitored, it can help determine the brain condition of an infant in emergencies where conventional EEG is not available and can provide information on decision making for cooling therapy in infant with $\mathrm{HIE}^{22}$ In cases of encephalopathy or abnormal tracing, it provides useful information for follow-up plans, evaluation of clinical improvement, the response to anticonvulsant. Actually, the number of aEEG exam is higher than the number of exams of brain USG, brain MRI, and cEEG. One cause of this observation was because in the case of immediate death in an urgent situation, further evaluation could not be performed. In other words, aEEG was useful to assess the patient even in emergency situation where other tests were not possible. In addition, initial assessment with aEEG monitoring helped to identify the patient who need to perform additional, tests that require sedation, such as brain MRI.

The enrolled patients were presented as seizures, seizurelike events, decreased activities, apnea or mental change. Some infants were taken aEEG monitoring because of increased seizure risk, such as hypocalcemia, or the need for background monitoring. More than half of them showed abnormal tracing on aEEG monitoring. Although aEEG is known to underestimate seizure than $\mathrm{CEEG}^{3,23}$ in this study, there were fewer cases of seizure on cEEG because it was not immediately available and was often performed after treatment and clinical improvement. Twenty-five percent of patients suspected of seizure could not observe seizures. One possibility of these dissociation is that it was not seizure but paroxysmal event. It could be seizures, but aEEG could not detect seizures because of improvement of seizure after anticonvulsant medication. aEEG could be difficult to detect seizures at different sites besides central or parietal areas because only a small area of the brain surface is covered by limited channel electrodes. A majority of neonatal seizures are relatively brief and missed even on aEEG monitoring. ${ }^{15}$
Therefore, aEEG should be assessed comprehensively with other evaluation and the physicians should not judge solely based on aEEG.

In fact, subtle seizures have been the most frequent seizures in neonatal seizures. ${ }^{7}$ In this study, clonic seizures is the most frequently observed. In the case of clonic seizures, it is likely to be detected at electrodes on aEEG monitoring while involves the motor cortex. It has been shown that the majority of neonatal seizures originates in central and temporal areas. ${ }^{3,24,25}$ It should be noted that in some cases only electrical seizures were observed clinical seizures. Neonatal seizures may have electroclinical dissociation, and such electrical seizures should also be considered for treatment. Only electrical seizures without clinical seizures can be detected only by EEG monitoring, so aEEG, which can be used for real-time long-term monitoring, is suitable for NICU. In practice, when monitoring seizures only visually in NICU, it underestimates actual seizures. In this study, $13.0 \%$ of seizures was only electrical seizures without no clinical seizures. Even in infants with clinical seizures, not all seizures were detected by medical staffs. Although there are still pitfalls in the interpretation of the aEEG by unskilled staff and artifacts in NICU environment, ${ }^{26,27}$ the aEEG monitoring could improve optimal neonatal seizure management through revealing multiple clinically silent seizures and subclinical status epilepticus in highrisk infants. ${ }^{28}$

In this study, we analyzed later epilepsy in patients with aEEG monitoring and did not identify differences with respect to gender, delivery mode, or inborn/outborn status. In the context of abnormal aEEG tracing, infants had increased risk of later epilepsy. A total of 25 of 71 infants with abnormal aEEG died, and there were no deaths among newborns with normal aEEGs. In fact, the ORs for death or later epilepsy with an abnormal $\mathrm{aEEG}$ increased to 15.7. In other words, the higher likelihood of epilepsy in infants with abnormal aEEG tracing compared with those with normal aEEG tracing suggest that they need constant follow-up.

The limitation of this study is that it is a retrospective study based on medical records and cannot be generalized in a heterogeneous patient groups. We did not separate the premature infants and term infants in etiology analysis. Moreover, the test was not performed as a routine for premature infants who have a high risk of brain damage. The use of aEEG monitoring for 
improving outcome in high-risk infants has not been directly proven. This study did not evaluate the prognostic value of aEEG monitoring for developmental outcomes other than later epilepsy. The aEEG would probably underestimate the seizure burden when compared to cEEG. Of note, interrater reliability for subjective aEEG interpretation is not perfect. ${ }^{29}$ Nonetheless, it is always feasible, even if other tests are not available, and easy to run and easy to read in the NICU setting. It is also suitable for long-term surveillance of cerebral activities of newborns. If it used in conjunction with other monitoring techniques, it could obviously provide additional information about the infant's cerebral condition to aid patient management. ${ }^{3,30}$

In conclusion, this study confirms observations that aEEG monitoring is useful and well-tolerated techniques for cerebral monitoring in NICU. The aEEG monitoring enable to assess the cerebral integrity of the infant through background activities, recognize seizures earlier, detect subclinical seizures, to help more precise management including anticonvulsant therapy and cooling and evaluate the clinical improvement and response to medication.

\section{Conflict of interest}

No potential conflict of interest relevant to this article was reported.

\section{References}

1) Maynard D, Prior PF, Scott DF. A continuous monitoring device for cerebral activity. Electroencephalogr Clin Neurophysiol 1969;27:672-3.

2) Viniker DA, Maynard DE, Scott DF. Cerebral function monitor studies in neonates. Clin Electroencephalogr 1984;15:185-92.

3) Hellström-Westas L. Amplitude-integrated electroencephalography for seizure detection in newborn infants. Semin Fetal Neonatal Med 2018; 23:175-82

4) Shah DK, Mackay MT, Lavery S, Watson S, Harvey AS, Zempel J, et al. Accuracy of bedside electroencephalographic monitoring in comparison with simultaneous continuous conventional electroencephalography for seizure detection in term infants. Pediatrics 2008;121:1146-54.

5) Shah NA, Wusthoff CJ. How to use: amplitude-integrated EEG (aEEG). Arch Dis Child Educ Pract Ed 2015;100:75-81.

6) Jensen FE. The role of glutamate receptor maturation in perinatal seizures and brain injury. Int J Dev Neurosci 2002;20:339-47.
7) Kanhere S. Recent advances in neonatal seizures. Indian J Pediatr 2014 81:917-25

8) Papile LA, Burstein J, Burstein R, Koffler H. Incidence and evolution of subependymal and intraventricular hemorrhage: a study of infants with birth weights less than 1,500 gm. J Pediatr 1978;92:529-34.

9) de Vries LS. Neurological assessment of the preterm infant. Acta Paediatr 1996;85:765-71.

10) Tatli B, Guler S. Non epileptic paroxysmal events in childhood. Turk Pediatri Ars 2017;52:59-65.

11) Thoresen $M$, Hellström-Westas $L$, Liu $X$, de Vries LS. Effect of hypothermia on amplitude-integrated electroencephalogram in infants with asphyxia. Pediatrics 2010;126:e131-9.

12) Toet MC, Hellström-Westas L, Groenendaal F, Eken P, de Vries LS. Amplitude integrated EEG 3 and 6 hours after birth in full term neonates with hypoxic-ischaemic encephalopathy. Arch Dis Child Fetal Neonatal Ed 1999;81:F19-23.

13) al Naqeeb N, Edwards AD, Cowan FM, Azzopardi D. Assessment of neonatal encephalopathy by amplitude-integrated electroencephalography. Pediatrics 1999;103(6 Pt 1):1263-71.

14) Abend NS, Wusthoff CJ. Neonatal seizures and status epilepticus. Clin Neurophysiol 2012;29:441-8.

15) Clancy RR, Legido A, Lewis D. Occult neonatal seizures. Epilepsia 1988; 29:256-61.

16) Gluckman PD, Wyatt JS, Azzopardi D, Ballard R, Edwards AD, Ferriero $D M$, et al. Selective head cooling with mild systemic hypothermia after neonatal encephalopathy: multicentre randomised trial. Lancet 2005; 365:663-70.

17) Shalak LF, Laptook AR, Velaphi SC, Perlman JM. Amplitude-integrated electroencephalography coupled with an early neurologic examination enhances prediction of term infants at risk for persistent encephalopathy. Pediatrics 2003;111:351-7.

18) Azzopardi D; TOBY study group. Predictive value of the amplitude in tegrated EEG in infants with hypoxic ischaemic encephalopathy: data from a randomised trial of therapeutic hypothermia. Arch Dis Child Fetal Neonatal Ed 2014;99:F80-2.

19) Hellström-Westas L, Klette H, Thorngren-Jerneck K, Rosén I. Early predic tion of outcome with aEEG in preterm infants with large intraventricular hemorrhages. Neuropediatrics 2001;32:319-24.

20) Hellström-Westas L, Rosén I, De Vries LS, Greisen G. Amplitude-integrated EEG classification and interpretation in preterm and term infants. NeoReviews 2006;7:e76-87.

21) Hayakawa M, Okumura A, Hayakawa F, Watanabe K, Ohshiro M, Kato Y, et al. Background electroencephalographic (EEG) activities of very preterm infants born at less than 27 weeks gestation: a study on the degree of continuity. Arch Dis Child Fetal Neonatal Ed 2001;84:F163-7.

22) Sarkar S, Barks JD, Donn SM. Should amplitude-integrated electroencephalography be used to identify infants suitable for hypothermic neuroprotection? J Perinatol 2008;28:117-22.

23) Shellhaas RA, Soaita Al, Clancy RR. Sensitivity of amplitude-integrated electroencephalography for neonatal seizure detection. Pediatrics 2007;120:770-7 
24) Bourez-Swart MD, van Rooij L, Rizzo C, de Vries LS, Toet MC, GebbinkTA, et al. Detection of subclinical electroencephalographic seizure patterns with multichannel amplitude-integrated EEG in full-term neonates. Clin Neurophysiol 2009;120:1916-22.

25) Biagioni E, Frisone MF, Laroche S, Kapetanakis BA, Ricci D, Adeyi-Obe M, et al. Maturation of cerebral electrical activity and development of cortical folding in young very preterm infants. Clin Neurophysiol 2007; 118:53-9.

26) ter Horst HJ, van Olffen M, Remmelts HJ, de Vries H, Bos AF. The prognostic value of amplitude integrated EEG in neonatal sepsis and/or meningitis. Acta Paediatr 2010;99:194-200.
27) Toet MC, Lemmers PM. Brain monitoring in neonates. Early Hum Dev 2009:85:77-84.

28) Hellström-Westas $L$, Rosén I, Swenningsen NW. Silent seizures in sick infants in early life. Diagnosis by continuous cerebral function monitoring. Acta Paediatr Scand 1985;74:741-8.

29) Shellhaas RA, Gallagher PR, Clancy RR. Assessment of neonatal electroencephalography (EEG) background by conventional and two amplitude-integrated EEG classification systems. J Pediatr 2008;153:369-74.

30) Gunn JK, Beca J, Hunt RW, Olischar M, Shekerdemian LS. Perioperative amplitude-integrated EEG and neurodevelopment in infants with congenital heart disease. Intensive Care Med 2012;38:1539-47. 